\title{
INFLUÊNCIA DA IDADE NO TEMPO DE INTERNAÇÃO E NO GRAU EVOLUTIVO DAS APENDICITES AGUDAS
}

\author{
AGE INFLUENCE IN HOSPITAL STAY LENGTH AND ACUTE APENDICITIS EVOLUTION \\ GRADE
}

\author{
Marcelo Wilson Rocha Almeida ${ }^{1}$; Ângela Trevisan João ${ }^{1}$; Fernanda Silveira de Oliveira ${ }^{2}$; \\ Humberto Carvalho de Mattos ${ }^{3}$; André Rodrigues da Silva ${ }^{4}$; Maria Cristina G. Barbosa e Silva ${ }^{5}$
}

\begin{abstract}
RESUMO: Objetivos: Comparar o grau evolutivo da apendicite determinada pela inspeção trans-operatória com o resultado anatomopatológico, e identificar alguma relação entre a idade, grau evolutivo da apendicite aguda e o tempo de internação hospitalar. Método: Análise retrospectiva dos prontuários de 199 pacientes submetidos a apendicectomia entre o período de outubro de 2003 a agosto de 2004, quanto à idade, sexo, tempo de internação e a fase do processo inflamatório segundo anatomopatológico das peças. Resultados: A análise foi possível em 182 casos. Nestes pacientes, a distribuição quanto ao sexo mostrou maior incidência em homens, com 54,4\% casos; a mediana da idade foi de 20 anos, sendo a faixa etária mais prevalente entre os 11-20 anos com $36,22 \%$ dos casos. A mediana do tempo de internação foi de três dias. Houve uma diferença significativa do tempo de internação de acordo com a faixa etária dos pacientes, sendo que os pacientes acima de 60 anos tiveram maior tempo de internação. O anatomopatológico evidenciou $73,62 \%$ casos de apendicite supurada, $13,73 \%$ apendicite branca, $7,14 \%$ gangrenosa, $4,49 \%$ catarral e $0,54 \%$ neoplásico, confirmando o diagnóstico de apendicite em $86,24 \%$. O tempo de internação e a idade não foram significativamente diferentes entre os graus evolutivos da apendicite aguda. Conclusão: A apendicite aguda ocorre com maior freqüência nos pacientes jovens e do sexo masculino. Os pacientes idosos permanecem mais tempo internados, porém não houve diferença na idade nem tempo de internação em relação aos vários graus evolutivos da apendicite (Rev. Col. Bras. Cir. 2006; 33(5): 294-297).
\end{abstract}

Descritores: Apendicite; Doença aguda; Tempo de internação; Prognóstico.

\section{INTRODUÇÃO}

A apendicite aguda é a doença mais freqüente do apêndice vermiforme e a principal causa de cirurgia abdominal de urgência, sendo a apendicectomia a primeira escolha para seu tratamento ${ }^{1-3}$. Sua incidência é maior em jovens em idade escolar e adolescência, predominando o sexo masculino e a raça branca ${ }^{1}$.

Pode manifestar-se em diferentes fases: catarral, supurada e gangrenosa ${ }^{4}$. Estudos mostram que está relacionada a um pior prognóstico em pacientes idosos e com doenças associadas, assim como um maior tempo de evolução da doença e à ocorrência de perfuração apendicular- ${ }^{5-9}$.

O presente estudo tem por objetivo identificar uma possível relação entre a idade, o grau evolutivo da apendicite aguda e o tempo de internação hospitalar dos pacientes. Também será comparado o grau evolutivo da apendicite determinado pela inspeção trans-operatória com o resultado anatomopatológico das respectivas apendicectomias.

\section{MÉTODOS}

Entre outubro de 2003 e agosto de 2004 foram realizadas 199 (cento e noventa e nove) apendicectomias no Hos- pital Universitário "São Francisco de Paula", em Pelotas, RS. A partir deste grupo, procedeu-se a análise retrospectiva do prontuário dos pacientes quanto à idade, sexo, tempo de internação hospitalar e grau evolutivo da apendicite, segundo inspeção trans-operatório e avaliação anatomopatológica. Para este fim, as peças cirúrgicas foram classificadas em apendicite catarral aguda (CTA), supurada (SUP), gangrenosa (GAN) e incluídas as formas branca (BRA) e neoplásica (NEO).

O estudo foi submetido ao Comitê de Ética em Pesquisa e aprovado com louvor. Os dados obtidos tiveram dupla digitação e foram validados através do programa EPI-INFO, versão 6. Para análise dos dados utilizouse o programa Stata $9.2 \AA$, com realização de análise de variância (ANOVA) para avaliar diferenças de idade entre as categorias do grau de evolução da apendicite e do tempo de internação entre categorias de faixas etárias (por décadas) e graus de evolução. Foi realizada a estatística de kappa para avaliar o grau de concordância entre a inspeção trans-operatória e o diagnóstico anatomopatológico, sendo considerado boa a concordância para kappa $>0,60$. Para a significância estatística, foi considerado um valor $\mathrm{p}<0,05$.

\footnotetext{
1. Médico-Residente em Cirurgia Geral do Hospital Universitário São Francisco de Paula (HUSFP) da Universidade Católica de Pelotas - UCPel.

2. Médica-Residente em Pediatria do Hospital Universitário São Francisco de Paula ( HUSFP) da Universidade Católica de Pelotas - UCPel.

3. Cirurgião Geral do HUSFP da Universidade Católica de Pelotas -UCPel.

4. Acadêmico da Escola de Medicina da Universidade Católica de Pelotas (UCPel).

5. Professora Adjunta da Clínica Cirúrgica da Escola de Medicina da UCPel.
}

Recebido em 24-07-2006

Aceito para publicação em 30-08-2006

Conflito de interesses: nenhum

Fonte de financiamento: nenhuma

Trabalho realizado no Serviço de Cirurgia Geral do Hospital Universitário São Francisco de Paula de Pelotas, RS. 


\section{RESULTADOS}

Do total de 199 (Cento e noventa e nove) pacientes do período estudado, 17 foram excluídos do estudo por apresentarem prontuários incompletos. Da amostra final de 182 (cento e oitenta e dois) pacientes, 99 (54,4\%) pertenciam ao sexo masculino. A idade variou entre 2 e 77 anos, sendo a média de 20 anos. A faixa etária mais prevalente foi entre os pacientes de 11 a 20 anos (36,22\%), seguida da faixa de 21 as 30 anos (20,54\%). (Gráfico 1).

Metade dos pacientes (91) permaneceu três dias internada (mediana), variando entre 2 a 15 dias (Gráfico 2). Houve uma diferença significativa do tempo de internação de acordo com a faixa etária dos pacientes, sendo que os pacientes acima de 60 anos tiveram maior tempo de internação $(p<0,001)$ (Gráfico 3). Embora tenha sido encontrado um maior tempo de internação na fase gangrenosa com 4,62 dias, esta não se mostrou estatisticamente significativa $(p=0,09)($ Gráfico 4). Também não foi encontrada diferença entre a idade média e as diferentes fases evolutivas da apendicite aguda $(p=0,7)$, sendo a maior idade média encontrada na fase catarral $(29 \pm 12,5$ anos).

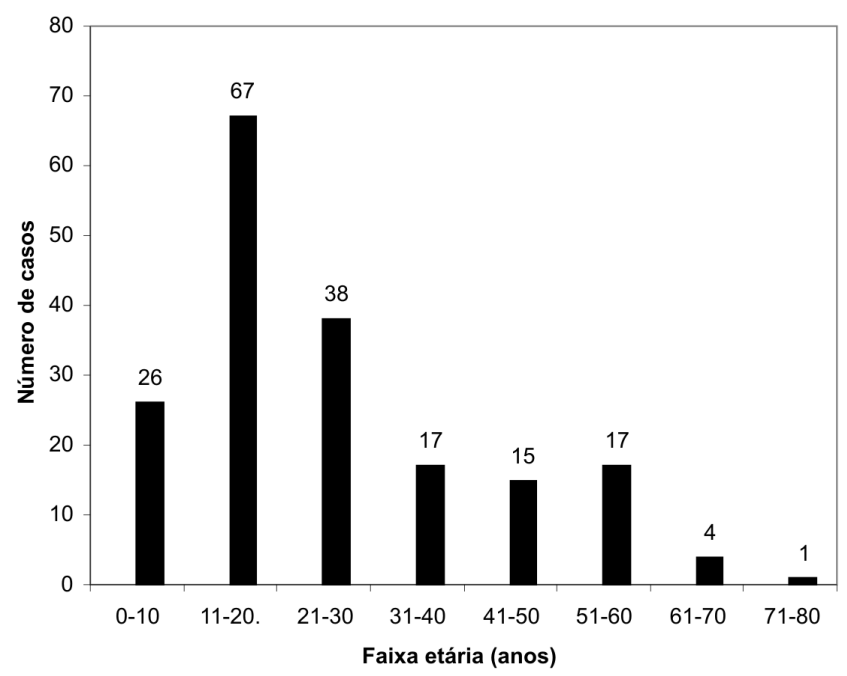

Gráfico 1 - Distribuição da freqüência de casos de apendicite segundo a faixa etária em anos.

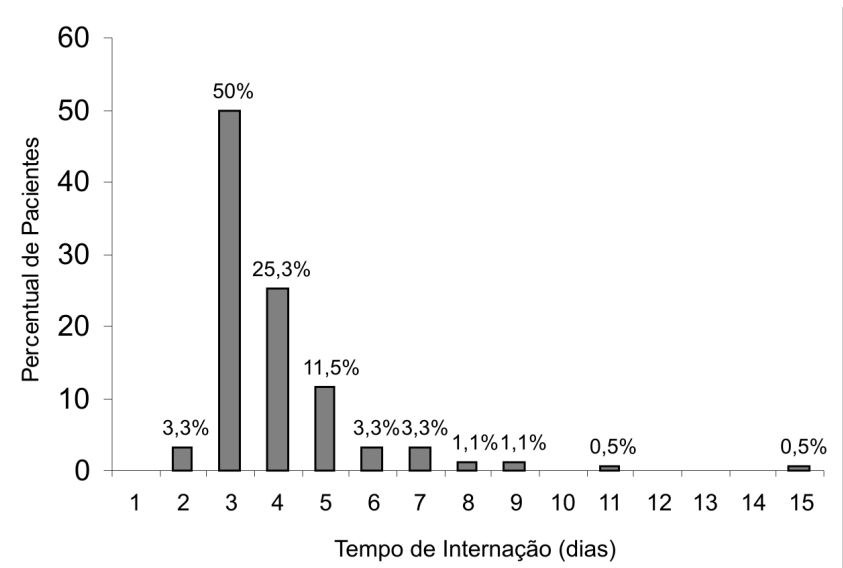

Gráfico 2 - Distribuição da freqüencia de pacientes segundo tempo de internação em dias.
A Tabela 1 mostra a concordância do grau evolutivo no momento da inspeção trans-operatória com o anatomopatológico. Foi encontrada uma concordância fraca entre as duas avaliações (kappa $=0,18)$.

\section{DISCUSSÃO}

A apendicite aguda é uma doença extremamente freqüente, com formas de apresentação diversas e a causa mais comum de cirurgia abdominal de urgência ${ }^{5}$. Incide, principalmente, na faixa etária jovem, mas não raramente em idosos, sendo mais prevalente no sexo masculino ${ }^{2,5,9}$. Em nosso estudo, $54,4 \%$ dos pacientes eram do sexo masculino e a grande maioria (70,4 \%) tinham menos de 31 anos de idade. Esses dados condizem com os encontrados na literatura.

Diversos estudos demonstram que a apendicite aguda em idosos está associada a um maior grau de complicações. Redmond et al. ${ }^{6}$, analisando 126 (cento e vinte e seis) casos de apendicite aguda concluiu que os idosos apresentam um prognóstico mais grave para a doença devido a maior freqüência de apendicite perfurado. Gurleyik ${ }^{5}$, em um estudo avaliando 109 (cento e nove) pacientes com mais de 50 anos,

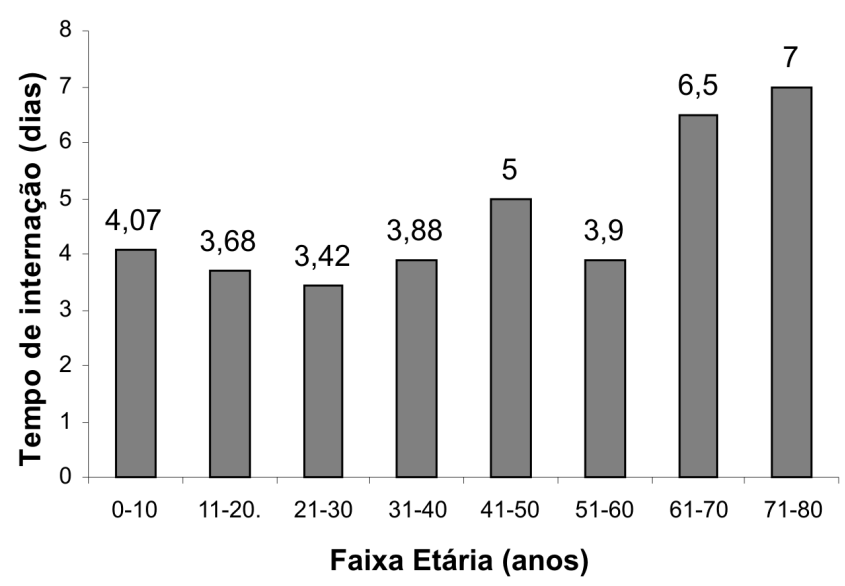

Gráfico 3 - Tempo médio de internação (em dias) segundo a faixa etária (em anos) $(p<0,001)$.

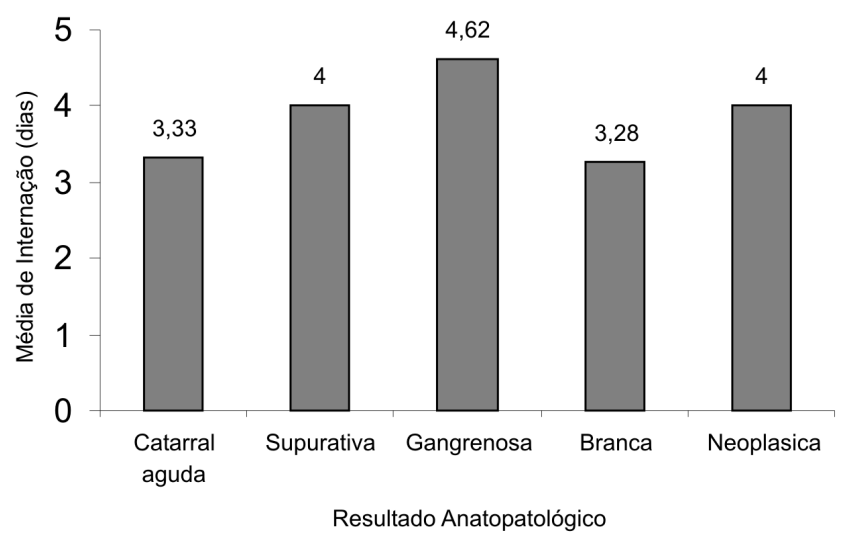

Gráfico 4 - Tempo médio de internação (em dias) segundo grau evolutivo da apendicite aguda $(p=0,09)$. 
Tabela 1 - Comparação da inspeção trans-operatória com o diagnóstico anatomopatológico (concordância $($ kappa $)=0,18)$.

\begin{tabular}{|c|c|c|c|c|c|c|c|}
\hline \multirow{2}{*}{$\begin{array}{l}\text { Anatomo } \\
\text { patológico }\end{array}$} & \multirow[b]{2}{*}{ SUP } & \multicolumn{5}{|c|}{ Inspeção trans-operatória } & \multirow[b]{2}{*}{ Total } \\
\hline & & CTA & GAN & & EO & BRA & \\
\hline SUP & $67 \quad(50 \%)$ & $26(19 \%)$ & $36 \quad(27 \%)$ & 3 & $(2,3 \%)$ & $2(1,5 \%)$ & 134 \\
\hline CTA & $2 \quad(22,2 \%)$ & $7(77,8 \%)$ & $0 \quad(0 \%)$ & 0 & $(0 \%)$ & $0 \quad(0 \%)$ & 9 \\
\hline GAN & $6 \quad(46,1 \%)$ & $1 \quad(7,7 \%)$ & $6 \quad(46,1 \%)$ & 0 & $(0 \%)$ & $0 \quad(0 \%)$ & 13 \\
\hline NEO & $1(100 \%)$ & $0 \quad(0 \%)$ & $0 \quad(0 \%)$ & 0 & $(0 \%)$ & $0 \quad(0 \%)$ & 1 \\
\hline BRA & $4 \quad(16 \%)$ & $15(60 \%)$ & $0 \quad(0 \%)$ & 0 & $(0 \%)$ & $6(24 \%)$ & 25 \\
\hline Total & $80 \quad(44 \%)$ & $49(27 \%)$ & $42 \quad(23 \%)$ & 3 & $(1,65 \%)$ & $8 \quad(4,4 \%)$ & 182 \\
\hline
\end{tabular}

CTA: Catarral Aguda; SUP: Supurativa; GAN: Gangrenosa; BRA: Branca; NEO: Neoplasia.

encontrou uma taxa de perfuração significativamente mais elevada em idosos que pacientes pediátricos e adultos jovens, bem como aumento da morbidade e mortalidade nos idosos com perfuração do apêndice. Pittman-Waller et al. ${ }^{10}$, analisando 5755 (cinco mil setecentos e cinqüenta e cinco) casos, verificou incidência de apendicectomias complicadas de $32 \%$, sendo maior em pacientes do sexo masculino e em idade avançada. Em nosso estudo, os pacientes da faixa etária de 61 a 70 anos tiveram uma média de 6,5 dias de internação e, os de 71 a 80 anos, média de sete dias, sendo significativamente superior às demais faixas etárias $(\mathrm{p}<0,001)$. Sugerimos que os pacientes idosos necessitam de maior ênfase e atenção, pois neste grupo o diagnóstico é freqüentemente retardado e as complicações são mais comuns. No entanto, não encontramos neste estudo uma associação entre a maior gravidade (fases supurativas e gangrenosas) e a idade $(p=0,7)$, sendo a maior idade média encontrada nos pacientes com apendicite na fase catarral.

Em nosso estudo, foi encontrada uma taxa significativa de 134 (cento e trinta e quatro) casos $(73,62 \%$ de apêndices) em estágio supurativo e 13 casos $(7,14 \%)$ em estágio gangrenoso, significando, portanto, mais de $80 \%$ de apendicites complicadas. O tempo médio de internação, apesar de ser diferente entre os diversos estágios evolutivos, não apresentou significância estatística $(p=0,09)$. Este achado difere dos resultados encontrados no estudo de Fischer', que analisou 272 (duzentos e setenta e dois) pacientes submetidos a apendicectomia, encontrando média de internação de 3,8 dias para apendicite não complicada e cinco dias para os casos complicados.
Observou-se uma fraca concordância entre o grau evolutivo no momento da inspeção trans-operatória com o anatomopatológico (kappa $=0,18$ ). A melhor concordância ocorreu na fase catarral, com 77,8\% dos casos, sendo todas as demais fases subestimadas. Este dado é relevante, pois a indicação do uso de antibióticos, seja de maneira profilática ou terapêutica, baseia-se na avaliação realizada na inspeção trans-operatória, podendo assim modificar a evolução da apendicite ${ }^{11-13}$.

O diagnóstico de apendicite aguda é eminentemente clínico, baseado nos sinais e sintomas, sendo importante o conhecimento destes para uma indicação cirúrgica correta. Em nosso estudo, foi confirmando o diagnóstico de apendicite em $86,24 \%$ semelhante ao encontrado no estudo de Ricci et al. ${ }^{14}$, que avaliou 295 (duzentos e noventa e cinco) casos de apendicectomia e encontrou precisão diagnóstica de $81,4 \%$. Sugere-se a implantação de diretrizes para diagnóstico e tratamento de apendicite aguda, para que ocorra a cirurgia em fases mais precoces e que o tempo de internação seja o menor possível. A busca pelo conhecimento dos sinais e sintomas e da inspeção trans-operatória das apendicites pelos cirurgiões tem que ser constante, para que, a exemplo de outros estudos, se obtenha um aumento na confirmação diagnóstica ${ }^{15}$.

Conclui-se que a apendicite aguda ocorre com maior freqüência nos pacientes jovens e do sexo masculino. Os pacientes idosos permanecem mais tempo internados, porém não houve diferença na idade, nem no tempo de internação em relação aos vários graus evolutivos da apendicite. Apesar de ser a causa mais comum de abdome agudo de urgência a apendicite necessita de maior ênfase no seu diagnóstico e tratamento para que os pacientes permaneçam o menor tempo possível internados.

\footnotetext{
ABSTRACT

Background: To study appendicitis evolution grade in comparison with intraoperative inspection and pathological results. Also a relationship between age and hospital stay length is reviewed. Method: Retrospective analysis of 199 patients submitted to appendicectomy between October 2003 through August 2004 using: age, sex, length of hospital stay and evolution grade of inflammatory process according to pathological examination was studied. Results: Analysis was possible only in 182 cases. Distribution by sex showed a higher incidence in men (54.4\%); median age was 20 years and the most prevalent age range was between $11-20$ years $(36.22 \%)$. Hospital median stay length was 3 days. There was a significant difference in hospital stay length according to age and patients older than 60 years; they had the longest hospital stay length. Pathologic examination showed $73.62 \%$ as suppurative appendicitis, normal appendix was $13.73 \%$, gangrenous was $7.14 \%$, catarrhal was $4.49 \%$ and neoplasm was found in $0.54 \%$. Diagnosis of appendicitis was confirmed in $86.24 \%$. Hospital stay length and age were not significant different between acute appendicitis evolution grades. Conclusion: Acute appendicitis is more frequent in young male patients. Older patients stay longer in hospital; however appendicitis evolution grades showed neither correlation with age nor length of stay.
}

Key words: Appendicitis; Acute disease; Length of stay; Prognosis. 


\section{REFERÊNCIAS}

1. Fitz RH. Perforating inflammation of the vermiform appendix with special reference to its early diagnosis and treatment. Am J Med Sci. 1886;92:321-46.

2. Addis DG, Shaffer N, Fowler BS, Tauxe R. The epidemiology of appendicitis and appendectomy in the United State. Am J Epidemiol. 1990;132(5):910-25.

3. Wolkomir A, Kornak P, Elsakr M, McGovern P. Seasonal variation of acute appendicitis: a 56-year study. South Med J. 1987;80(8):958-60.

4. Cotran RS, Kumar V, Robbins SL, editors. Robbins: patologia estrutural e funcional. Rio de Janeiro: Guanabara Koogan; 2000.

5. Gurleyik G, Gurleyik E. Age-related clinical features in older patients with acute appendicitis. Eur J Emerg Med. 2003;10(3):200-3.

6. Redmond JM, Smith GW, Wilasrusmee C, Kitturs DS. A new perspective in appendicitis: calculation of half time $(\mathrm{T}(1 / 2))$ for perforation. Am Surg. 2002; 68(7):593-7.

7. Watters JM, Blakslee JM, March RJ, Redmond ML. The influence of age on the severity of peritonitis. Can J Surg. 1996;39(2):142-6.

8. Franz MG, Norman J, Fabri PJ. Increased morbidity of appendicitis with advancing age. Am Surg. 1995;61(1):40-4.

9. Fischer CA, Pinho MSL, Ferreira S, Milani CAC, van Santen CR, Marquardt RA. Apendicite aguda: existe relação entre o grau evolutivo, idade e o tempo de internação? Rev Col Bras Cir. 2005;32(3):136-8.

10. Pittmann-Waller VA, Myers JG, Stewart RM, Dent DL, page CP, Gray GA, Pruitt BA Jr, Roof HD. Appendicitis: why so complicated? Analysis of 5755 consecutive appendectomies. Am Surg. 2000;66(6):548-54.
11. Ricci MA, Trevisani MF, Beck WC. Acute appendicitis. A 5year review. Am Surg. 1991;57(5):301-5.

12. Lunca S, Bouras G, Romedea NS. Acute appendicitis in the elderly patient: diagnostic problems, prognostic factors and outcomes. Rom J Gastroenterol. 2004; 13(4):299-303.

13. Valle Espinoza A. Antibióticos en apendicitis aguda. Managua 2004 Feb:51.

14. Newman K, Ponsky T, Kittle K, Dyk L, Throop C, Gieseker K, Sills M, Gilbert J. Appendicitis 2000: variability in practice, outcomes, and resource utilization at thirty pediatric hospitals. J Pediatr Surg. 2003;38(3):372-9.

15. Emil S, Laberge JM, Mikhail P, Baican L, Flageole H, Nguyen L, Sahw K. Appendicitis in children: a ten-year update of therapeutic recommendations. J Pediatr Surg. 2003;38(2):236-42.

Como citar este artigo:

Almeida MWR, Trevisan AJ, Oliveira FS, Mattos HC, Silva AR, Barbosa e Silva MCG. Influência da idade no tempo de internação e grau evolutivo das apendicites agudas: uma revisão em um Hospital Universitário do sul do país. Rev Col Bras Cir. [periódico na Internet] 2006 Set-Out;33(5). Disponível em URL: www.scielo.br/rcbc

Endereço para correspondência:

Marcelo Wilson Rocha Almeida.

Rua Santos Dumont, 548, apto 301, Ed. Camila.

96020-380 - Pelotas, RS.

Telefone: xx (53) 3225-7151.

E-mail: mwralmeida@brturbo.com.br. 